\section{Wnt/FZD Genetics in Glioblastoma}

\section{Sergio Casas-Tintó*}

Instituto Cajal, CSIC, Madrid, Spain

\section{Introduction}

Wnt/FZD signalling pathway regulates proliferation, migration, cell fate and death. Deregulation of this pathway is one of the hallmarks for glioblastoma (GBM), the most malignant brain tumour. Wnt/FZD aberrant signalling promotes glioma cell proliferation, propagation and resistance to conventional chemo and radiotherapy. Current research efforts to understand the signalling deregulation by $W n t / F Z D$ focus on the design of Wnt inhibitors and on the characterization of downstream effectors. However, little is known about the origin of this deregulation. To date, there is no evidence for gain of expression of Wnt or its receptors even though downstream effectors are clearly upregulated in GBM. Among the novel alternatives we discuss here the downregulation of Wnt antagonists and the deregulation of vesicle trafficking system in GBM cells. Both strategies would lead to an oncogenic trigger towards increasing Wnt signalling.

\section{Comment and Discussion}

The origin of mutations in specific genes has been essential to decipher the cause and progression of human diseases. The primitive idea of one-gene-one protein has evolved during the last decades and, nowadays, the term genetic mutation uncovers a range of possibilities from single base pair changes in the open reading frame to epigenetic modifications of regulatory regions. The latest has emerged as a revolutionary explanation for a number pathological scenario in which no Mendelian mutations are detected in the open reading frame of the gene but gene deregulation is evident.

We have recently reviewed the contribution of Wnt to central nervous system diseases [1]. In particular, we have addressed WNT contribution in two of the most common pathologies of the CNS, Alzheimer's disease and Glioblastoma (GBM).

In the canonical pathway, Wnt is activated upon binding to specific receptors in the plasma membrane (LRPs and FZD). Then, a cascade of events occurs in the cytoplasm of the cell which includes the inactivation of a destruction complex (APC and Axins) that releases $\beta$-catenin (armadillo in Drosophila) and its subsequent translocation into the nucleus. There, $\beta$-cat stimulates the expression of a collection of genes (i.e., Cycling D1 and Myc) that drive changes in cell proliferation and physiology.

GBM is a type of brain tumor originated in glial cells, astrocytes. It is characterized for its aggressiveness and great infiltrative nature. GMB is resistant to the standard treatments including tumor resection, radiotherapy and chemotherapy (Temozolomide). Thus, few patients survive longer than 5 years after diagnosis [2] and the average survival rate is shorter than 2 years [3].

One of the hallmarks of bad prognosis in GMB is the activation of $\beta$-cat [4], indicating an over-activation of the WNT/FZD pathway [5]. Following that rationale, we searched in the COSMIC database (Collection of Somatic Mutations in Cancer) for gain of function mutations or copy number variations in $W n t / F Z D$ genes. The analysis showed that mutations in Wnt or FZD are rather sporadic and do not correlate with GBM progression. These data are consistent with the lack of mutations in $\beta$-catenin in GBM patients [6].

This finding might result contradictory since the over-activation of this pathway has been well documented in several types of cancer

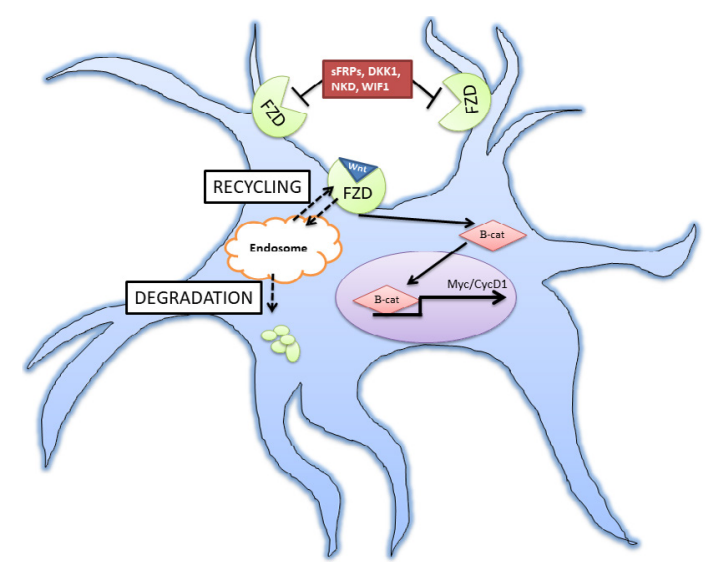

Figure 1: Schematic representation of a GBM cell. Wnt/FZD signaling regulates $\beta$-catenin translocation into the nuclei to promote gene expression. Pathway agonists expression (sFRPs, DKK1, NKD or WIF1) is downregulated in GBM cells facilitating Wnt signaling. FZD receptor is internalized and then either recycled or sent to degradation, the equilibrium of this endosomal system is altered in GBM cells and can mediate Wnt signaling through FZD stability.

including GBM [4]. On the other hand, it seems clear that $\beta$-catenin plays a central role in other types of human cancers [7-9]. Recent studies have focused in other components of the pathway and their contribution to cancer development. For example, APC, $\beta$-catenin, AXIN, WTX and TCF4 also contribute to the development of certain types of cancer $[10,11]$.

Alternative strategies could account for pathway activation without up regulating Wnt or FZD. Recent studies have proposed epigenetic modifications as an intriguing alternative for regulating the activity of signalling pathways. In particular, sFRPs, Dickkopf, NKD and WIF1 are potent inhibitors of the Wnt signal transduction pathway which happen to be down regulated in GBM [12] (Figure 1). Deregulation of these proteins account for $40-50 \%$ of primary GBM cases [12,13]. Epigenetic changes including promoter hypermethylation reduce the expression of these inhibitors and, as a consequence, there is a hyperactivation of $W N T / F Z D$ signalling. In turn, this effect yields to $\beta$-cat activation in GBM cells and contributes to tumor progression (reviewed in [14]).

Likely, however, the number of strategies to activate WNT pathway in GBM cells is still incomplete. Among the additional possibilities to account for the WNT pathway activation in the absence of genomic mutations or epigenetic modifications, cellular mechanisms favouring oncogenic signalling have been proposed recently for GBM. In particular, endosomal trafficking which mediates receptor internalization, signalling, recycling and proteolysis is deregulated in GBM.

*Corresponding author: Casas-Tintó S, Instituto Cajal, CSIC, Madrid, Spain, Tel: 91585 4750; E-mail: scasas@cajal.csic.es

Received July 10 2017; Accepted July 31, 2017; Published August 07, 2017

Citation: Casas-Tintó S (2017) Wnt/FZD Genetics in Glioblastoma. Hereditary Genet 6: 186. doi:10.4172/2161-1041.1000186

Copyright: @ 2017 Casas-Tintó S. This is an open-access article distributed under the terms of the Creative Commons Attribution License, which permits unrestricted use, distribution, and reproduction in any medium, provided the original author and source are credited. 
Wnt/FZD and Epidermal Growth Factor Receptor (EGFR) pathways are pivotal in GBM progression and interactions between these signals are well documented [15]. Vesicle trafficking regulates Receptor Tyrosine Kinase (RTK) pathways such as EGFR [16] as well as others including Wnt pathway $[17,18]$. This novel perspective would fit with the lack of receptor expression upregulation but, with an increase in $\beta$-catenin activity (Figure 1). The validation of this approach is currently under study, endosomal trafficking needs to be established as a primary or secondary cause of oncogenesis in GBM.

\section{Conclusion}

The genetic heterogeneity and rapid transformation of GBM cells grants resistance to current therapeutical treatments. There is a need to design novel strategies against oncogenic signals in GBM which cannot rely on founder mutations. Cellular characteristics such as receptor recycling if shared by GBM cells, are not necessarily genetic mutations. These tumor features emerge as objects of study and the genes implicated might be of interest for future therapies. Wnt/FDZ pathway is critical for tumoral growth and has appeared as a novel feature in GBM. Despite the lack of genetic mutations driving Wnt/FZD overexpression, downstream targets upregulation is a hallmark in patients. We discuss other possibilities besides genetic mutations in oncogenes as a strategy to increase Wnt pathway signaling. Downregulation of Wnt antagonists or over activation of the endosomal recycling system may possibly increase Wnt signaling and promote oncogenic growth in GBM cells.

\section{References}

1. Arnes M, Casas Tinto S (2017) Aberrant Wnt signaling: A special focus in CNS diseases. J Neurogenet p. 1-7.

2. Stupp R, Hegi ME, Mason WP, Van Den Bent MJ, Taphoorn MJ, et al. (2009) Effects of radiotherapy with concomitant and adjuvant temozolomide versus radiotherapy alone on survival in glioblastoma in a randomised phase III study: A 5-year analysis of the EORTC-NCIC trial. Lancet Oncol 10: 459-466.

3. Sacko A, Hou MM, Temgoua M, Alkhafaji A, Marantidou A, et al. (2015) Evolution of the Karnosky performance status throughout life in glioblastoma patients. J Neurooncol 122: 567-573.
4. Sareddy GR, Panigrahi M, Challa, S, Mahadevan A, Babu PP (2009) Activation of Wnt/beta-catenin/Tcf signaling pathway in human astrocytomas. Neurochem Int 55: 307-317.

5. Rheinbay E, Suva ML, Gillespie SM, Wakimoto H, Patel AP, et al. (2013) An aberrant transcription factor network essential for Wnt signaling and stem cell maintenance in glioblastoma. Cell Rep 3: 1567-1579.

6. Reya T, Clevers $\mathrm{H}$ (2005) Wnt signalling in stem cells and cancer. Nature 434 843-850.

7. De Sousa EMF, Vermeulen $L$ (2016) Wnt Signaling in cancer stem cell biology. Cancers (Basel) 8: 1.

8. Suwala AK, Hanaford A, Kahlert UD, Maciaczyk J (2016) Clipping the wings of glioblastoma: Modulation of WNT as a novel therapeutic strategy. J Neuropathol Exp Neurol 75: 388-396.

9. Xue G, Romano E, Massi D, Mandala M (2016) Wnt/beta-catenin signaling in melanoma: Preclinical rationale and novel therapeutic insights. Cancer Treat Rev 49: 1-12.

10. Polakis $P$ (2012) Wnt signaling in cancer. Cold Spring Harb Perspect Biol 4.

11. Chiurillo MA (2015) Role of the Wnt/beta-catenin pathway in gastric cancer: An in-depth literature review. World J Exp Med 5: 84-102.

12. Foltz G, Yoon JG, Lee H, Ma L, Tian Q, et al. (2010) Epigenetic regulation of wnt pathway antagonists in human glioblastoma multiforme. Genes Cancer 1: 81-90.

13. Roth W, Wild-Bode C, Platten M, Grimmel C, Melkonyan HS, et al. (2000) Secreted frizzled-related proteins inhibit motility and promote growth of human malignant glioma cells. Oncogene 19: 4210-4220.

14. Lee $\mathrm{Y}$, Lee JK, Ahn SH, Lee J, Nam DH (2016) WNT signaling in glioblastoma and therapeutic opportunities. Lab Invest 96: 137-150.

15. Paul I, Bhattacharya S, Chatterjee A, Ghosh MK (2013) Current understanding on EGFR and Wnt/beta-Catenin Signaling in glioma and their possible crosstalk. Genes Cancer 4: 427-446.

16. Miaczynska M (2013) Effects of membrane trafficking on signaling by receptor tyrosine kinases. Cold Spring Harb Perspect Biol 5: a009035.

17. Clague MJ, Liu H, Urbe S (2012) Governance of endocytic trafficking and signaling by reversible ubiquitylation. Dev Cell 23: 457-467.

18. Gross JC, Chaudhary V, Bartscherer K, Boutros M (2012) Active Wnt proteins are secreted on exosomes. Nat Cell Biol 14: 1036-1045. 